ARID International Journal for Science and

Technology (AIJST)

ISSN: 2662-009X

Journal home page: http://arid.my/j/aijst

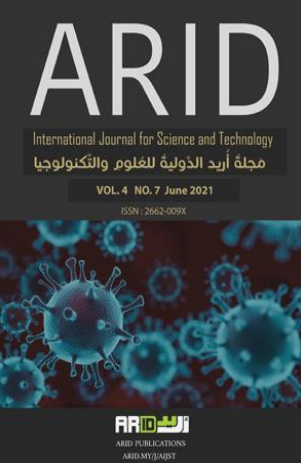

$$
\text { مَجلةُ أُريد الدَّوليةُ للعُلورج والتّكنولوجيا }
$$

\title{
Traditional Radiation Therapy for Treatment Breast Cancer and Radiation Effects on Normal Tissues
}

\author{
Abbood Abbas Abbood \\ International Sakharov Environmental Institute of Belarusian State University (ISEI BSU) \\ 23/1 Dolgobrodskaya Street, Minsk, 220070, Belarus \\ Belarusian/Minsk
}
العلاج الإثعاعي التقليدي لعلاج مريضات سرطان الثدي وتأثير جرعات الإشعاعية على الأنسجة الطبيعة$$
\text { عبود عباس عبود }
$$$$
\text { جامعة بيلاروسيا الحكومية -بيلاروسيا/مينسك }
$$

abbood.abbas.abbood@gmail.com arid.my/0004-6707

https://doi.org/10.36772/arid.aijst.2021.475 


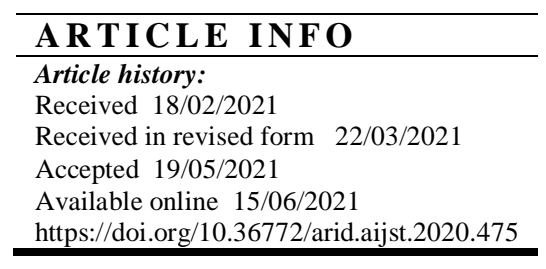

\begin{abstract}
The study aims to investigate the effect of traditional radiotherapy on cancer cells and normal cells near the tumor. This study aims to investigate recent advances in radiation therapy using high-energy targeted radiation doses for tumors with low radiation effects on normal cells near tumors or without radiological effects on normal cells. A study of five breast cancer patient's was taken. Three breast cancer patients had treatment by traditional radiation therapy. The other two patients were treated with modern radiation therapy. Traditional radiation therapy can affect patients with breast cancer in areas such as the spinal cord, heart, liver, and lungs. Treating the right side of the breast will impact the patient's liver and right lung. Treating the left side of the breast will have an impact on the heart and left side of the lung.
\end{abstract}

Keywords: Radiation therapy, cancer breast, lung, heart, spinal cord.

This paper is a revised and expanded version of a paper entitled 'Traditional radiation therapy for treatment breast cancer and radiation effects on normal tissues' presented at Fourth (1 international) student conference veterinary medicine and sustainable development goals, Egypt, 9 March 2021. 


\section{الملخص}

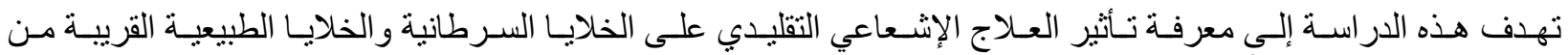

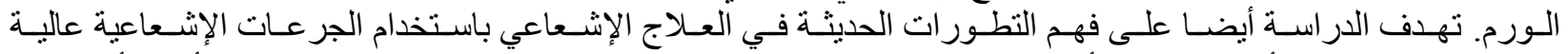

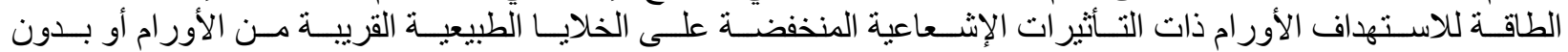

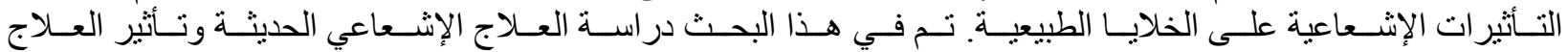

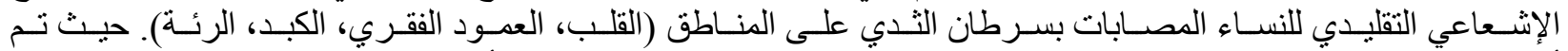

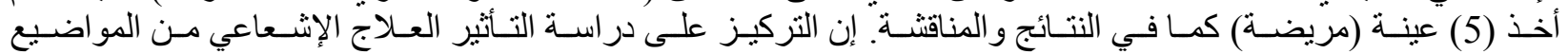

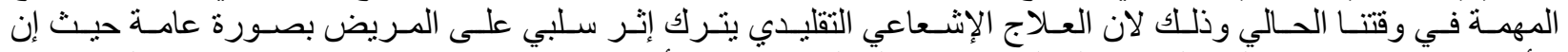

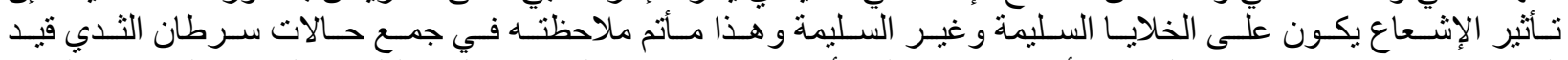

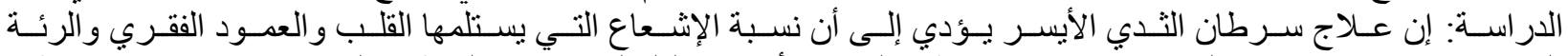

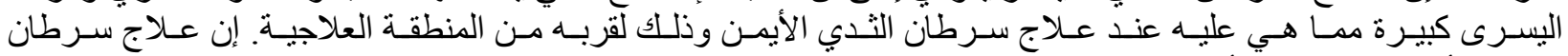

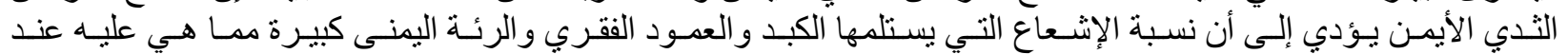

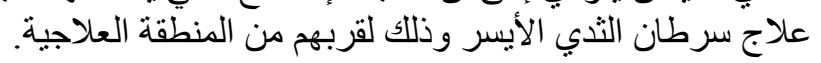

كلمات مفتاحية: العلاج الإشعاعي التقليدي، سرطان الثذي، القلب، الرئة. 


\section{1- Introduction:}

Traditional radiation therapy is the use of high-energy x-rays to destroying cancer cells but depending on the radiation doses it can have an effect on healthy cells. Traditional radiation therapy is often the most common radiation treatment but there are additional treatments that are also used to treat cancer cells such as surgery and/or chemotherapy. With traditional radiation therapy the division of the number of sessions, allows healthy cells to recover and repair themselves which contributes to cut the side effects of this type of treatment. Traditional radiation therapy sends $\mathrm{x}$-rays or photon beams to the cancerous tumor but it can also send these beams beyond the tumor which will then affect healthy tissues nearby and can cause substantial side effects $[1,2]$.

Nowadays, with the advances of radiation therapy have today using (proton therapy, brachytherapy, or treatment planning. etc.) able to send radiation doses of high-energy on cancer cells that stops at the tumor, making it less likely to affect healthy tissues that are near the tumor. Recent advancements have improved efficacy, reduced risks, and decrease the implications of radiation therapy. These advances involve Volumetric Modulated Arc Therapy (VMAT), Three-Dimensional Conformal Radiation Therapy (3DCRT), Stereotactic Radiotherapy (SRT), Brachytherapy, Intensity-Modulated Radiation Therapy (IMRT), Nano Therapy, RadioImmunotherapy (RIT), and others. Each of these modalities is the improved radiation targeting to a tumor, thereby limiting radiation exposure to healthy tissues. The modalities also deliver a lethal dose of radiation to the tumor cells. The use of recent advances in the treatment planning process distinguishes it from other forms of traditional radiotherapy of the high-resolution tumor-focused radiation dose delivery plan. As a result, the higher and more effective dose for the radiation is delivered 
directly to cancerous cells. At the same time, this greatly decreases the high dosage of radiation on the healthy tissues surrounding them $[1,3,4]$.

The current problem, to date, is the use of traditional radiation therapy in the treatment of cancerous cells and using the high dosage of radiation that kills the cancerous cells but has an effect on normal cells. With recent advancements in methods of radiation therapy for cancerous cell, these new methods reduce the effects of radiation doses on normal cells that are near the tumor. The objective of this study is to use methods of radiation therapy for the treatment of cancer cells using the most recent advancements of devices for radiation therapy.

The study aims to identify the most improved and efficient procedure, reduced risk, reduction of radiation therapy side-effect, and the best radiation methods that target only the tumor. The study also aims to provide the best methods of high beam doses on tumors with low side effects on normal cells.

Among the study problems presented by the current research on the questions main.

Some define certain sub-questions:

What is traditional therapy?

What is radiation therapy?

What are the methods of radiation therapy for cancerous cells?

The importance of the current study stems from the fact that it's recent studies on the effects of radiation doses on healthy cells and the importance in:

\section{Rational}

\section{Theoretical aspect}

The current research is considered one of the studies that accompany continuous studies. It is important to keep pace with developments in cancer cell therapies, in general. We must always consider the continuation of previous research and studies in 
cancer cell treatments, keeping in mind the importance of radiation therapy for the treatment of cancerous cells is a modern and advanced process.

\section{Practical aspect}

Adding and presenting scientific results that are of interest to researchers in the fields of treatments for cancer cells.

\section{2- Material and Methods}

The methods of patient's treatment of breast cancer by traditional radiation therapy and radiation therapy.

\section{2-1 Radiation Therapy}

The last studies on radiation therapy for treating breast cancer and research were discovered a century ago. One of the most common and effective uses in cancer treatments is radiotherapy. Generally, radiation doses of high energy are used to destroy cancer cells and shrink tumors. Types of radiotherapy include Brachytherapy and external beam radiation. Brachytherapy (internal radiation therapy) is a type of radiation therapy that involves inserting seeds, ribbons, or capsules containing a source of radiation into or near the tumor. To destroy cancer cells and shrink tumors. The most prevalent type of radiotherapy is external beam radiation. This includes an external beam therapy that guides radiation doses of high energy on cancer cells from outside the body and radiation from a variety of different angles that reaches the tumor. Radiation therapy is more effective in radiation with different intensities, the aim is for radiation doses to focus on tumor locations without causing damage to the normal tissue. Using radiation therapy for the treatment of almost all types of cancer $[2,3,4]$. 


\section{2-2 Breast Cancer}

Breast cancer is a condition in which out-of-control cells develop in the breast. Different kinds of breast cancer are present. The type of breast cancer depends on which cells transform into cancer in the breast. Breast cancer may start in various parts of the breast. Many tumors are benign in the breast, but certain types of abnormal cells may begin to split out of control and become malignant. When a tumor is breast cancerous, it can spread from its first place to nearby tissues, and when the infection develops further. Cancer can reach the lymphatic vessels or into the bloodstream and this will allow cancer to spread to other places further in the body. Types of breast cancer include invasive ductal carcinoma and invasive lobules carcinoma. The cancer cells spreads into other areas of the breast tissue beyond the ducts. Invasive cancer cells may also spread to other areas of the body. There are also other less common types of breast cancer, such as medullary external symbol, Paget disease, breast cancer that is inflammatory, and mucinous [5,6].

Ductal carcinoma in situ (DCIS) is a breast illness that may lead to breast cancer. Cancer cells are only found in the lining of the ducts and have not spread on other breast tissue [5].

Staging breast cancer is a way of identifying the extent to which breast cancer has spread and the size of the tumor, whether it has spread to lymph nodes, or whether the tumor has spread to remote parts of the body. The TNM method is the most common instrument that uses in staging breast cancer [7]. These divisions generally depend on three factors which are the following:

- (T), describes the tumor size and any cancer spread to surrounding tissue

- $(\mathrm{N})$, describes cancer spread to nearby lymph nodes 
- $(\mathrm{M})$, describes metastasis (spread for cancer on other parts of the body). Staging helps diagnose where cancer is found, whether and/or where it has spread, and whether other areas of the body cancer treatment were performed. It depends on the type of breast cancer that has spread and how far. There are methods such as Surgery, Hormonal therapy, Chemotherapy, Radiation therapy, Biological therapy, and other treatment [7].

High beam radiation therapy is used to treat breast cancer in different situations, some of which can be mentioned as follows [5]:

- Radiation therapy has only been used to treat breast cancer.

- The use of radiation therapy can merge with surgery, chemotherapy, and other treatments to treat breast cancer.

- After breast surgery, the use of radiation therapy in this case aims to reduce the chance of the tumor appearing again in the breast or the lymph nodes.

- After mastectomy, especially if cancer has spread to the lymph nodes.

- The condition in which breast cancer spreads to other parts of the breast such as the brain or bones.

\section{3- Results and Discussion}

The treatment of breast cancer in this study was conducted by traditional radiation therapy and radiation therapy. A study was done to illustrate the effects of radiation on sensitive areas for breast cancer patients (liver, heart, lung, spinal cord). The study will explain cases to effect of traditional radiotherapy on healthy tissues and cancer cells. Using a radiation dosimeter in the treatment of the cancerous tumor and is device measures the dose uptake for ionization radiation and using the unit of measurement for the calculation amount for radiation a patient is subjected to is gray (GY). This is also known as centigray (CGY) and as shown below. 
Case 1: The patient with left breast cancer was treated with traditional radiotherapy and was diagnosed with $\mathrm{CT}$ scan and had stage breast cancer $\left(\mathrm{T}_{2} \mathrm{~N}_{0} \mathrm{M}_{0}\right)$. Using radiation doses done the total volume $(2358.05 \mathrm{cc})$, the minimum dose is $(0.0 \mathrm{CGY})$, the maximum dose is (4374.0CGY), the mean dose is (3012.0 CGY), cursor volume (76.79\%), and as shown in the graph. the number of sessions is 15 fraction (Fx), and the beam dose rate effect is larger on the left lung (476 CGY), heart (1056 CGY), beam dose rate effect is less on the right lung (19 CGY), and right side breast (27 CGY). Using a much higher beam dosage (GTV), (CTV), (PTV) as shown in the red color for killing the cancerous cells in the right breast causing a radiation effect on the healthy cells (from 100 volumes to 4000 doses). Note, a beam dose rate effect larger on the left lung as shown in the green color (from 100 volumes to 3600 doses), heart as shown in the blue color (from 100 volumes to 3600 doses), and spinal cord but less effect on the right lung as shown in the yellow color (from 100 volumes to 500 doses), and right breast as shown in the green color (from 100 volumes to 700 doses), and liver than beam dose as shown in figure 1 .

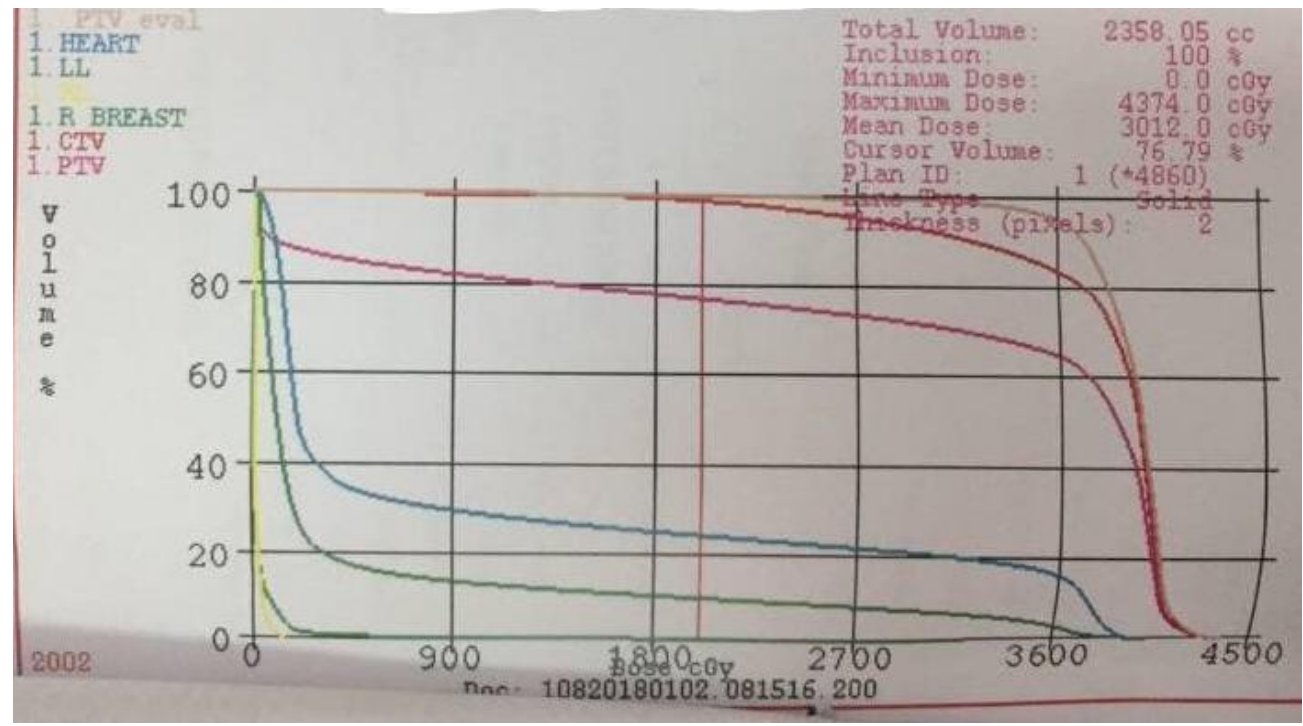

Figure(1): Graph volume-dose for treatment of left breast cancer 
Case 2: The patient with left breast cancer was treated with traditional radiotherapy and was diagnosed with $\mathrm{CT}$ scan and had stage breast cancer $\left(\mathrm{T}_{2} \mathrm{~N}_{2} \mathrm{M}_{0}\right)$. Using radiation doses done the total volume $(1527.33 \mathrm{cc})$, the minimum dose is $(173.0 \mathrm{CGY})$, the maximum dose is (4538.0 CGY), the mean dose is (3725.0 CGY), cursor volume $(97.95 \%)$, and as shown in the graph. The beam dose rate effects are larger on the left lung (1642 CGY), heart (671CGY). The beam dose rate has a less effect on the right lung (41CGY), and right side breast (10 CGY). Using high beam dosage for the killing of cancerous cells in the left breast causes a radiation effect on normal tissue at the same time (from 100 volumes to 4500 doses) as shown in the red. Note, a larger beam dose affects the left lung, heart, and spinal cord as shown in the red and yellow color but has a less effect on the right lung, liver, and right breast as shown in the blue color as shown in figure 2 .

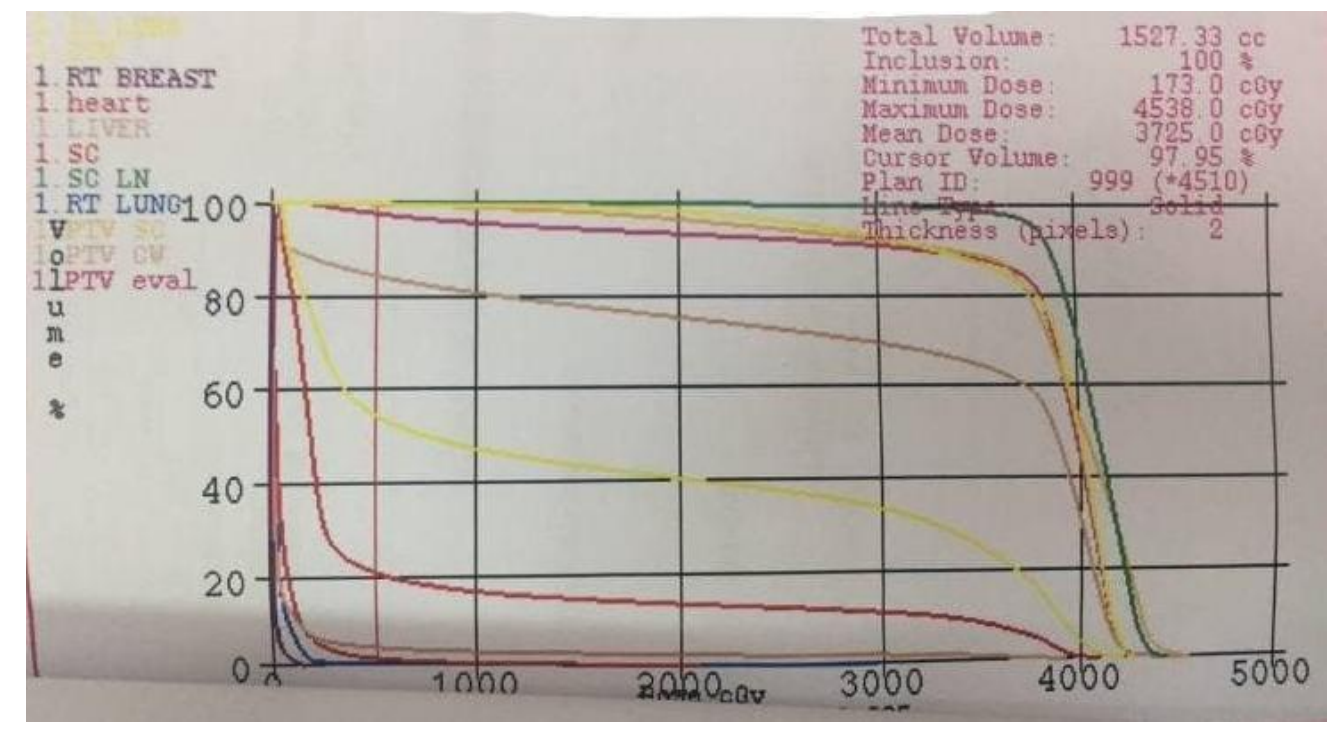

Figure(2): Graph volume-dose for treatment of left breast cancer by traditional radiation therapy 
Case 3: The patient with right breast cancer was treated with traditional radiotherapy and was diagnosed with $\mathrm{CT}$ scan and had stage breast cancer $\left(\mathrm{T}_{2} \mathrm{~N}_{1} \mathrm{M}_{0}\right)$. Using radiation doses done the total volume $(1233.20 \mathrm{cc})$, the minimum dose is (296.0 CGY), the maximum dose is (4322.0CGY), the mean dose is (3798.0 CGY), cursor volume (99.64\%), and as shown in the graph. Beam dose rate effect larger on right lung (1322 CGY), beam dose rate effect less on the heart (75 CGY), and left side breast (15 CGY) and left lung (23 CGY). Using a high beam dose for the killing cancerous cells in the right breast with radiation effect on healthy cells (from 100 volumes to 4050 doses). Note, a high beam dose rate effect is larger on the right lung as shown in the yellow color, liver as shown in the green color, and spinal cord as shown in the red color (from 100 volumes to 4500 doses) but less effect on the left lung as shown in the yellow color (from 100 volumes to 900 doses), the heart as shown in the red color (from 100 volumes to 1800 doses), and the left breast as shown in figure 3.

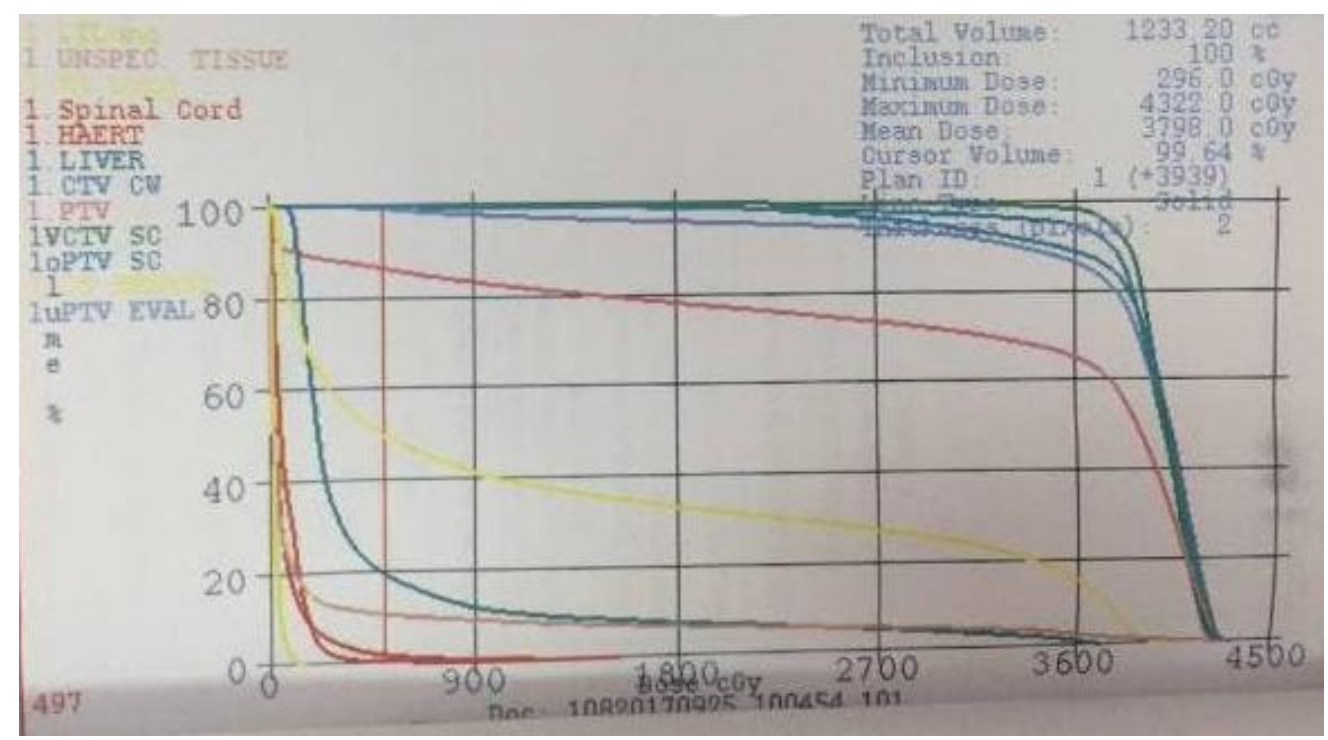

Figure(3): Graph volume-dose for treatment of right breast cancer 
Case 4: Post-mastectomy patient with radiotherapy of the right breast using a 6-domain sliding window for IMRT treatment for the treatment of supra / infraclavian and internal lymph nodes but with preservation of normal tissue from a high dose beam [3] as shown in figure 4.

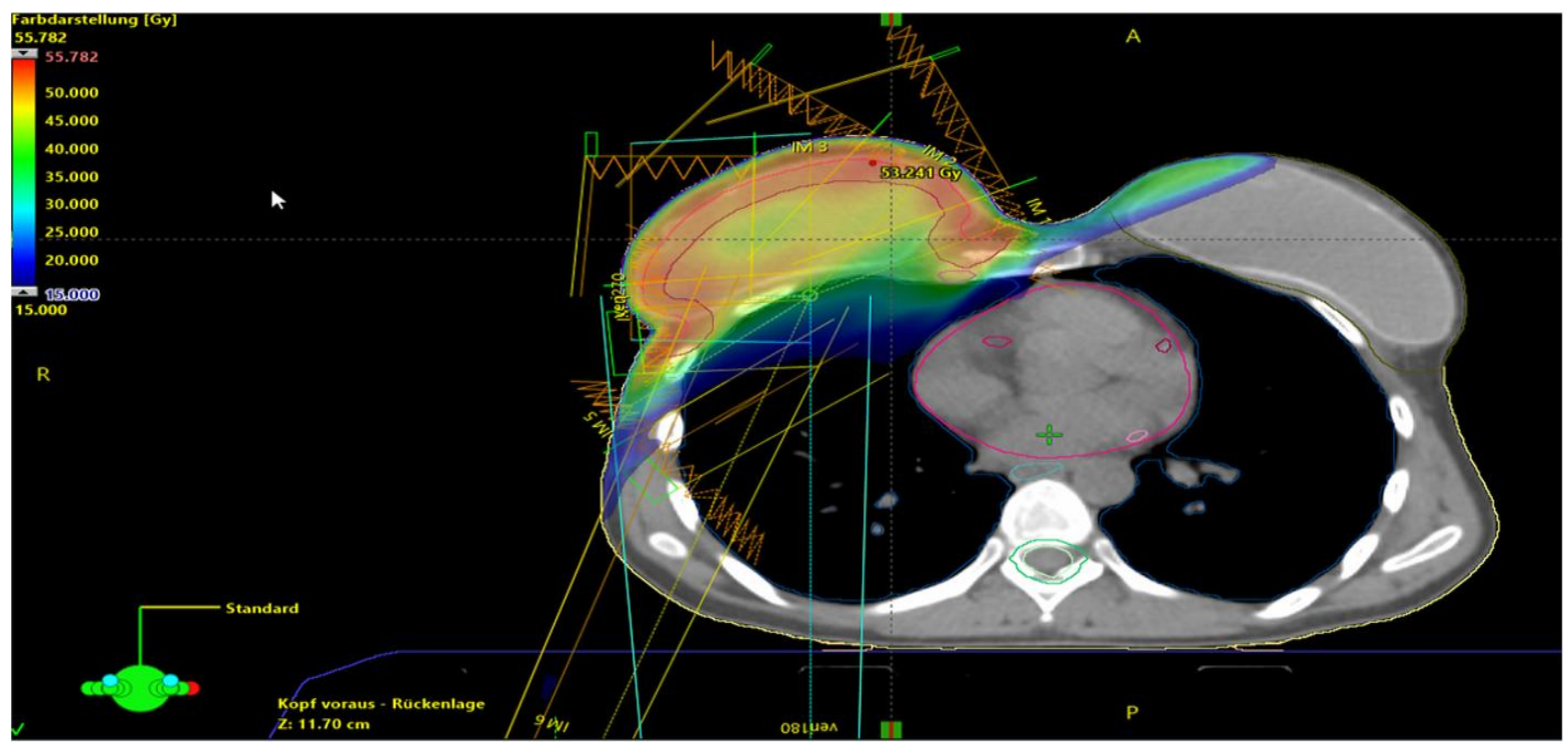

Figure(4): Right breast cancer patient [3]

Case 5: The patient was undergoing left breast radiation therapy using a 3-field sliding window IMRT technique. It is more effective, targets the tumor more directly and has

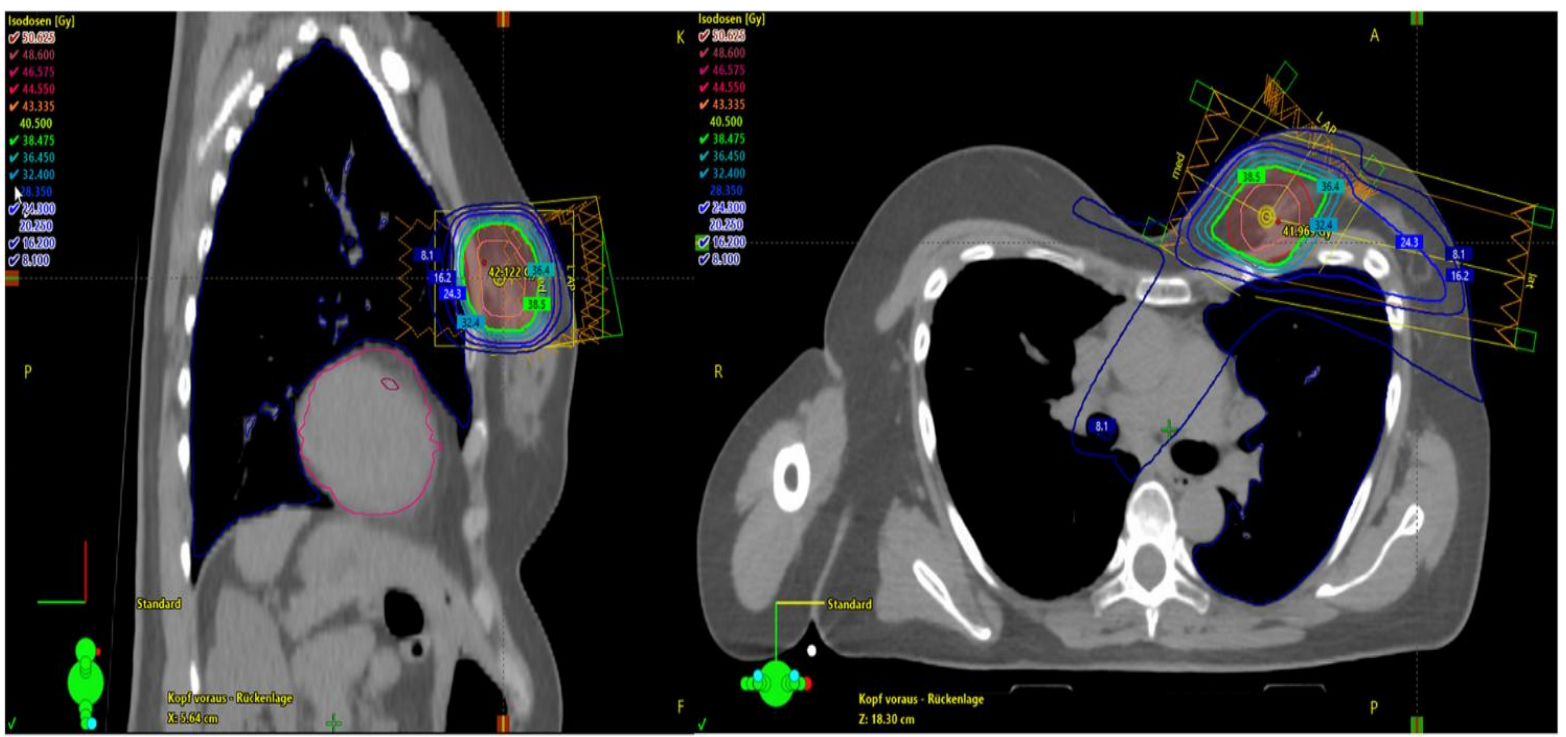

fewer side effects than traditional radiation therapy [3] as shown in figure 5.

Figure(5): Left breast cancer patient [3] 


\section{4- Conclusions:}

This study was done on the effect of traditional radiation therapy for breast cancer patients and on recent developments in radiation therapy that allows us to switch from two-dimensional to three-dimensional and four-dimensional imaging which provides more focused therapy. The study is intended to provide more reliable tumor radiation doses and biological targeting. This would cause the radiation dose to increase, thereby being more efficient at treating cancer and reducing the amount of time it takes to retreat. Radiation therapy has three cases:

The effect on the left breast on normal cells of traditional radiation therapy has a greater impact on the heart, spinal cord, and left lung when compared to the right side which has a less of an effect. The right side is less affected by traditional radiation therapy as in case 1 and in case 2 .

The effect of traditional radiation therapy on the right side breast on normal cells on the spinal cord, liver, and right lung is more than the left side, which is less affected by traditional radiation therapy as in case 3 .

Treating breast cancer using recent advances radiation therapy, proton therapy, brachytherapy, or using in treatment planning (3D, IMRT, VMAT, SIB, to other). These are perfect treatments for the patient's cancer. These methods kill cancerous cells with a more active radiation dose on the tumor with side-effects that are low on normal cells than with high dose beam as in the cases: case 5, case 6. 


\section{Recommendations}

I believe recent advances in radiation therapy are the alternative to traditional radiation therapy because it focuses more on removing the cancerous tumor with a less effect on healthy tissues.

\section{Abbreviations}

\begin{tabular}{|ll|ll}
\hline RT & Radiation therapy & RIT & Radio-immunotherapy \\
RTP & Radiation treatment planning & GTV & Gross tumor volume \\
IMRT & Intensity-modulated radiation therapy & CTV & Clinical target volume \\
VMAT & Volumetric modulated arc therapy & PTV & Planning target volume \\
3DCRT & Three-dimensional conformal radiation & CGY & Centigray \\
& therapy & FX & Fraction \\
SRT & Stereotactic radiotherapy & CC & Cubic centimeter \\
SIB & Simultaneous integrated boost & & \\
\hline
\end{tabular}




\section{References}

1. Issam El Naqa. A guide to outcome modeling in radiotherapy and oncology listening to the data. CRC Press. 1st edition. 2018.

2. Markus MacGill. what to know about radiation therapy? Medical news today. 2019 (accessed $10 \quad$ February 2021). https://www.medicalnewstoday.com/articles/158513.

3. J. Haussmann, S. Corradini, C. Nestle-Kraemling, E. Bölke, F. J. D. Njanang, B. Tamaskovics, k. Orth, E. Ruckhaeberle, T. Fehm, S. Mohrmann, I. Simiantonakis, W. Budach, C. Matuschek. Recent advances in radiotherapy of breast cancer. Radiation Oncology, 15(2020) 1-10.

4. C. Garibaldi, B. A. Jereczek-Fossa, G. Marvaso, S. Dicuonzo, D. P. Rojas, F. Cattani, A. Starzyńska, D. Ciardo, A. Surgo, M. C. Leonardi, R. Ricotti. Recent advances in radiation oncology. Ecancermedicalscience. 11(2017)1-19.

5. J. Strauss, W. Small, G. E. Woloschak. Breast cancer biology for the radiation oncologist. Springer. 2015.

6. Jaime Herndon. A Comprehensive Guide to Breast Cancer. Health line; 2019 (accessed 2 January 2021) https://www.healthline.com/health/breast-cancer.

7. A. E. Giuliano, J. L. Connolly, S. B. Edge, E. A. Mittendorf, H. S. Rugo, L. J. Solin, D. L. Weaver, D. J. Winchester, G. N. Hortobagyi. Breast Cancer-Major changes in the American Joint Committee on Cancer eighth edition cancer staging manual. A cancer journal for clinicians. Volume 67, Issue4(2017) 1-14. 